\title{
Ruta integral de atención en salud a través de la telesalud para el manejo del síndrome coronario agudo
}

\author{
Comprehensive health care route through telehealth for the management of acute \\ coronary syndrome
}

\author{
Diana C. Martínez-Pérez, Juan F. García-Cano y Carlos E. Vallejo-Bocanumen* \\ Grupo de Investigación en Urgencias y Emergencias (GIURE), Facultad de Medicina, Universidad de Antioquia, Medellín, Colombia
}

\section{Resumen}

Objetivo: Proponer una ruta integral de atención en salud a través de la telesalud para el manejo del síndrome coronario agudo. Método: La construcción de la ruta se basa en la revisión de la literatura y la información obtenida en grupos focales con expertos. Resultados: Se construyó una ruta integral de atención en salud para el manejo del paciente con síndrome coronario agudo. Se diagrama en notación gráfica que describe la lógica de pasos, teniendo en cuenta los siguientes actores: pacientes y familias, sistemas de atención de emergencias, telesalud, hospitales de primer y segundo nivel de complejidad, terceros niveles de complejidad con capacidad resolutiva de los casos de síndrome coronario agudo, y entes de inspección, vigilancia y control. Conclusiones: La ruta integral de atención en salud soportada en la telesalud es una apuesta innovadora para optimizar procesos en salud. Puede entenderse como alternativa ante la crisis que afronta el sistema en términos de financiamiento, resultados y legitimidad, teniendo en cuenta el efecto positivo de la telesalud sobre la oferta y el acceso a servicios, diagnósticos y tratamientos oportunos, la superación de distancias geográficas, el mejoramiento de la calidad y la contribución con el despliegue de las guías de práctica clínica.

Palabras clave: Ruta integral de atención en salud. Telesalud. Síndrome coronario agudo.

\section{Abstract}

Objective: To propose a comprehensive route of health care through telemedicine for the management of acute coronary syndrome. Method: The construction of the route is based on the literature review and the information obtained in focus groups with experts. Results: A comprehensive route of health care has been built for the management of patients with acute coronary syndrome. This comprehensive route of health care is diagrammed in a graphic notation that describes the logic of the steps, taking into account the following actors: the patient and their families, emergency care systems, telemedicine, first and second level of care hospitals, third levels of care with resolution capacity of acute coronary syndrome cases, and inspection surveillance and control authorities. Conclusions: The comprehensive route of health care supported in telemedicine, is an innovative bet to improve processes in health care services. It can be understood as an alternative to the crisis faced by the sector in terms of financing, results and legitimacy, taking into account the positive effect of telemedicine on the provision and access to services, diagnostic and timely treatment, to overcome geographic distances, improve quality and contribute to the deployment of clinical practice guidelines.

Key words: Local comprehensive health plans. Telemedicine. Acute coronary syndrome.

\section{Correspondencia:}

${ }^{*}$ Carlos E. Vallejo-Bocanumen

E-mail: eduardo.vallejo@udea.edu.co
Disponible en internet: 29-11-2021 Rev Colomb Cardiol. 2021;28(5):495-501 www.rccardiologia.com 0120-5633 / @ 2020 Sociedad Colombiana de Cardiología y Cirugía Cardiovascular. Publicado por Permanyer. Este es un artículo open access bajo la licencia CC BY-NC-ND (http://creativecommons.org/licenses/by-nc-nd/4.0/). 


\section{Introducción}

El síndrome coronario agudo es considerado una enfermedad crónica no transmisible. Es una enfermedad isquémica cardiaca y se produce por la obstrucción parcial o total de las arterias coronarias. Cada año causa la muerte de alrededor de 40 millones de personas en el mundo ${ }^{1,2}$. En Colombia es la principal causa de muerte en la población mayor de 55 años y explica el $49.5 \%$ de las muertes en el grupo de las enfermedades del sistema circulatorio y el $16.13 \%$ $(7,016,697)$ de todos los años de vida potencialmente perdidos, lo cual la convierte en un problema de salud pública ${ }^{3,4}$.

El Ministerio de Salud y Protección Social de Colombia, a través de las recomendaciones de la Guía de Práctica Clínica de Síndrome Coronario Agudo ${ }^{5}$ y otras guías internacionales de práctica clínica relacionadas $^{6-10}$, enfatiza la necesidad de realizar un manejo oportuno de los pacientes con síndrome coronario agudo, mediante estrategias diagnósticas, tratamiento farmacológico adecuado y técnicas de reperfusión temprana (apertura del vaso ocluido). Por lo tanto, es preciso identificar plenamente las estrategias de diagnóstico y tratamiento empleadas y disponibles en el país, así como garantizar el acceso a unos servicios de salud de calidad.

En Colombia se tiene claridad sobre las intervenciones eficaces para el manejo de pacientes con síndrome coronario agudo y sus indicaciones. Sin embargo, se presentan múltiples dificultades en su implementación, muchas de ellas relacionadas con factores individuales, clínicos, administrativos y del sistema de salud, tales como tipo de aseguramiento, concentración de especialistas y recurso tecnológico en centros urbanos, ubicación geográfica y nivel educativo de los pacientes y sus familiares, costos y gastos de bolsillo, entre otros, que se convierten en barreras de acceso a los servicios de salud ${ }^{11,12}$. Esto favorece el no cumplimiento del derecho a la salud, situación evidenciada en la persistencia de indicadores negativos de salud, lo cual sugiere fallas estructurales en el sistema ${ }^{13}$.

Con la finalidad de manejar los problemas señalados anteriormente, se genera un cambio del marco normativo expresado en la Ley Estatutaria en Salud 1751 que regula el derecho fundamental a la salud ${ }^{14} \mathrm{e}$ intenta mejorar el modelo de atención. De la misma forma, la Resolución 429 del año 2016 adopta la política de atención integral en salud y establece un modelo integral de atención en salud que tiene como finalidad la integralidad de la atención del paciente y derrumbar las barreras en la atención en salud ${ }^{15,16}$.

En este contexto, la telesalud aparece como una alternativa que facilita la superación de las barreras de acceso a los servicios de salud y permite mejorar la calidad de estos mediante el desarrollo de un conjunto de actividades, servicios y métodos diseñados para ser realizados a distancia, con la ayuda de las tecnologías de la información y las comunicaciones (TIC) ${ }^{17}$.

Crear rutas de atención enmarcadas en un nuevo modelo de salud, con directrices específicas soportadas en herramientas de telesalud, posibilita delimitar y optimizar procesos, minimizar gastos innecesarios de recursos y maximizar la ganancia en términos de indicadores de salud centrados en desenlaces de pacientes, eficiencia, eficacia, oportunidad y seguridad. Por estas razones, se evidencia la necesidad de desarrollar una ruta de atención integral en salud específica, encaminada a mejorar el acceso de la población con síndrome coronario agudo a intervenciones costo-efectivas, promoviendo la innovación y la consecución de los objetivos de desarrollo sostenible ${ }^{2}$.

\section{Método}

Para la construcción de la propuesta se llevó a cabo el siguiente proceso:

1) Revisión de la literatura especializada sobre telesalud y las guías de práctica clínica para síndrome coronario agudo. A partir de ella se referenció el marco normativo en Colombia, soportado en la Ley 1419 del año 2010, que tiene por objeto desarrollar la telesalud como apoyo al Sistema General de Seguridad Social en Salud.

2) Revisión del manual metodológico para la elaboración y la implementación de la ruta integral de atención en salud ${ }^{18}$, elaborado por el Ministerio de Salud y Protección Social de Colombia. Durante esta revisión se profundizó en las rutas integrales de atención específica para eventos, intervenciones individuales y colectivas dirigidas principalmente al diagnóstico oportuno, el tratamiento, la rehabilitación y la paliación de los eventos o las condiciones específicas de salud.

3) Desarrollo de un grupo focal al inicio del proceso, con la participación de cuatro profesionales de la salud: una médica general, un especialista en medicina de urgencias y dos residentes en medicina de urgencias. En este grupo se exploraron las dificultades en la atención, las experiencias y los 
posibles beneficios de las TIC para la prestación de servicios de salud.

4) Elaboración de la propuesta de ruta integral de atención en salud para síndrome coronario agudo a partir de la integración de las experiencias de los autores, en la que se diagramaron los momentos, los actores, los procesos, las tareas y los procedimientos que deben ocurrir para la atención en salud a partir de una ruta integral.

5) Desarrollo del segundo grupo focal con los integrantes mencionados en el primer grupo más dos subespecialistas en cardiología clínica, donde se hizo una validación de la propuesta de ruta integral de atención en salud. Para el desarrollo de los dos grupos focales la participación fue voluntaria, se recabó el consentimiento informado y se grabó con autorización de los participantes.

6) Realización de un tercer grupo focal con personal administrativo y asistencial de un centro hospitalario especializado de la ciudad de Medellín, donde se presentó la propuesta construida y se discutió acerca de la ruta integral de atención en salud construida hasta ese momento.

7) Modificaciones derivadas de los grupos focales y construcción de la propuesta final que aquí se presenta. Se modeló el diagrama en el software Biza$\mathrm{g}^{\circledR}{ }^{\circledR}$ que propone el Ministerio de Salud y Protección Social para la elaboración de rutas integrales de atención en salud ${ }^{18}$.

\section{Resultados}

La ruta integral de atención en salud para población con síndrome coronario agudo se diagrama (Fig. 1) en una notación gráfica que describe la lógica de los pasos, teniendo en cuenta los siguientes actores: el paciente y sus familias, los sistemas de atención de emergencias, la plataforma de telesalud, los hospitales de primer y segundo nivel de atención, los terceros niveles de atención con capacidad resolutiva de los casos de síndrome coronario agudo y los entes de vigilancia y control (entendidos como secretarías locales y departamentales de salud, y el Ministerio de Salud). En la figura 1 se especifican los momentos en los cuales interviene cada actor y las actividades que deben ser realizadas por cada uno de ellos para el desarrollo del caso. Se detallan las puertas de entrada a la ruta y se contemplan los momentos en que finaliza la atención del caso de síndrome coronario agudo.

La ruta integral de atención en salud del síndrome coronario agudo tiene un contenido clínico y otro administrativo. El contenido clínico permite guiar al médico tratante de una forma simplificada hacia momentos críticos para la toma de decisiones y la realización de intervenciones costo-efectivas basadas en las recomendaciones de la guía de práctica clínica para el manejo de los pacientes con síndrome coronario agudo. El componente administrativo permite clarificar y simplificar las actividades relacionadas con aseguramiento, referencia y contrarreferencia determinadas por la ley, que son críticas para el traslado efectivo a un centro hospitalario especializado para la resolución del caso. La plataforma tecnológica de telesalud realiza un acompañamiento clínico y un direccionamiento al personal del centro remisor por parte de personal idóneo del centro hospitalario especializado o de la misma plataforma, lo cual facilita la toma de decisiones y la realización de las intervenciones costo-efectivas consideradas por personal médico y esgrimidas en la guía de práctica clínica para la atención de la población con síndrome coronario agudo del Ministerio de Salud de Colombia. La asistencia de la plataforma tecnológica de telesalud se realiza de forma sincrónica o asincrónica, de acuerdo con el tipo de caso. Todos los casos de síndrome coronario agudo con elevación del ST tienen asistencia remota inmediata, y se determina la necesidad de reperfusión farmacológica in situ, así como intervenciones salvadoras de vida relacionadas con la particularidad del caso y el centro hospitalario especializado al cual debe llegar para su atención y resolución.

El prestador de servicios de salud que habilite un servicio de salud en la modalidad de telemedicina será responsable de, primero, cumplir con los estándares aplicables al servicio que se habilite, independientemente de que para su funcionamiento asistan diferentes organizaciones o personas para aportar al cumplimiento de los estándares, y segundo, garantizar la formación continua del talento humano en el manejo de la tecnología utilizada, en los procesos, los procedimientos y las herramientas inherentes a la prestación de los servicios, en la que se usen TIC. Las categorías de la telemedicina en Colombia se clasifican en telemedicina interactiva, telemedicina no interactiva, telexperticia y telemonitoreo ${ }^{19}$. La propuesta de una ruta integral de atención en salud del síndrome coronario agudo mediada por la telesalud se contempla mediante la telexperticia sincrónica, en la que intervienen dos profesionales de la salud, uno de los cuales atiende presencialmente al usuario y otro lo atiende a distancia. El profesional que atiende presencialmente al usuario es responsable del tratamiento, de las 


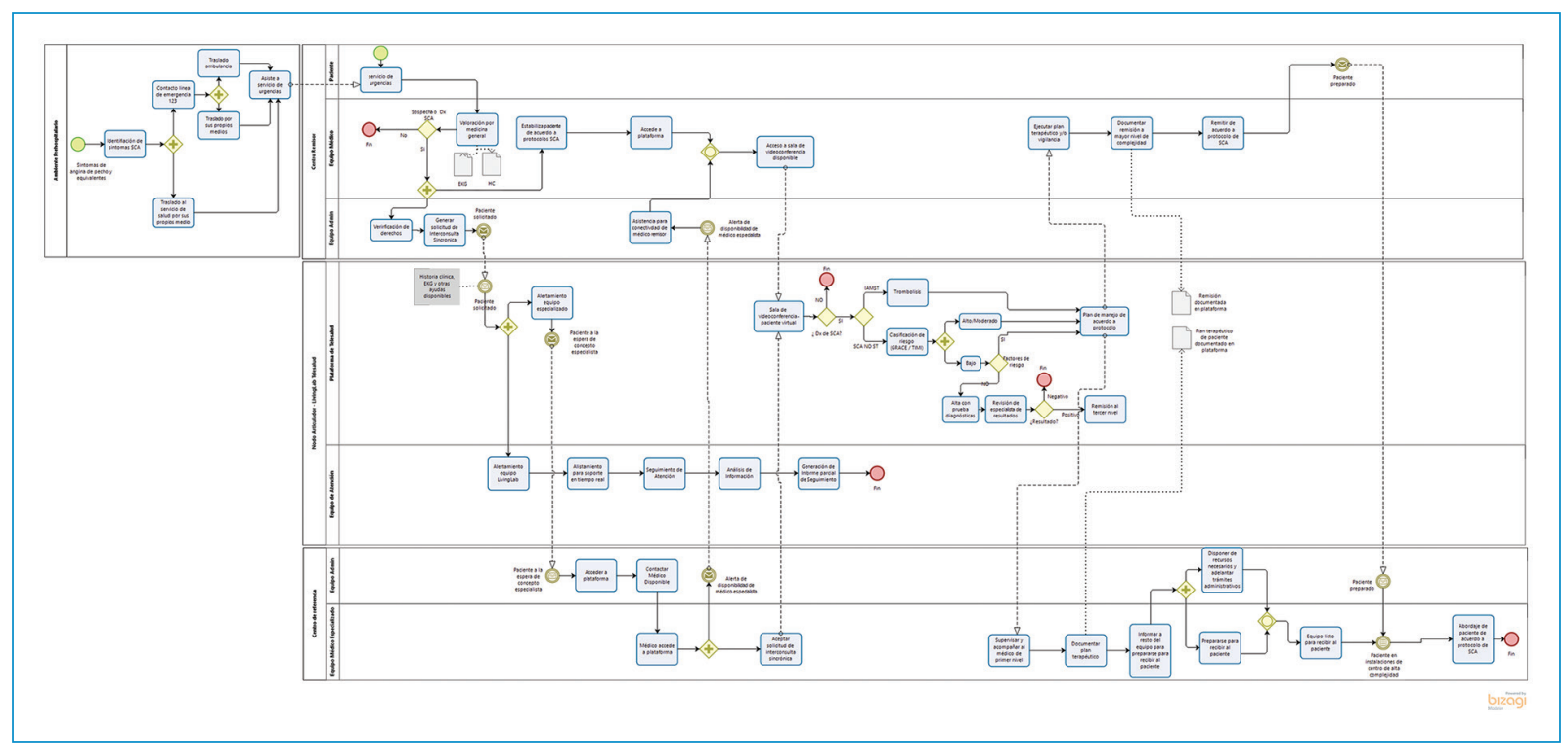

Figura 1. Modelo de ruta integral de atención para el manejo de la población con síndrome coronario agudo.

decisiones y recomendaciones entregadas al paciente, y el que atiende a distancia es responsable de la calidad de la opinión que entrega y tiene que especificar las condiciones en las que se da dicha opinión, lo cual debe consignarse en la historia clínica. Esta categoría está autorizada en Colombia para prescribir medicamentos por el profesional autorizado, y este será responsable de la prescripción que realice. La firma electrónica o la firma digital del profesional de la salud que se utilice en la prescripción tiene plena validez siempre y cuando cumpla los requisitos establecidos en la normativa vigente ${ }^{20}$.

Los centros hospitalarios especializados son parte esencial de la atención, dado que tienen la tarea de resolver los casos que les sean direccionados. Se propone que participen en la asistencia remota vía telesalud, como ya se mencionó, y se encarguen de realizar la atención clínica una vez que el paciente se encuentre en sus instalaciones. Por lo anterior, están interconectados con los primeros y segundos niveles de atención, y desarrollan la tarea de brindar concepto especializado durante los momentos en que el personal del centro remisor lo requiera. Se espera que la vinculación virtual al caso genere una mejoría en los ítems en la calidad de la atención y los desenlaces favorables.

La plataforma de telesalud permite el rastreo del caso y el registro de sus variables clínicas, sociodemográficas y administrativas que determinan las fortalezas y fallas en distintos momentos de la atención; esta información sirve como insumo para la evaluación caso a caso, y de forma macro, para análisis y toma de decisiones en la planeación de futuros cambios en los distintos niveles (estratégico, táctico u operativo).

A continuación, se presenta gráficamente la ruta integral de atención en salud del síndrome coronario agudo, cuya notación gráfica describe la lógica de los pasos del proceso (Fig. 1).

\section{Discusión}

La propuesta de una ruta integral de atención en salud del síndrome coronario agudo soportada en la telesalud es una apuesta innovadora de los procesos en los servicios de salud. Podría entenderse como una alternativa ante la actual crisis que afronta el sector en términos de financiamiento, resultados y legitimidad, al tomar en cuenta las posibilidades que tiene la telesalud para incidir sobre la oferta de servicios, mejorar la oportunidad en diagnósticos y tratamientos, superar distancias geográficas, facilitar el acceso a los servicios, mejorar la calidad y contribuir con el despliegue de las guías de práctica clínica ${ }^{21}$. Esta propuesta de ruta integral va acorde con lo planteado por el Ministerio de Salud de Colombia; es un modelo conceptual, construido desde la orientación de guías de práctica clínica de síndrome coronario agudo, nacionales e internacionales, y de los diferentes actores que participan en el proceso ${ }^{18}$. Se caracteriza por: 
- Describir explícitamente los elementos clave de la atención basada en la evidencia, las mejores prácticas y las expectativas de los usuarios.

- Permitir la integración organizada, mediante la secuenciación de las acciones multidisciplinarias de índole poblacional, colectiva e individual, y las funciones de los diferentes integrantes del Sistema de Seguridad Social en Salud y de las demás entidades que tengan a su cargo acciones en salud, en el marco de sus competencias y funciones.

- Fortalecer la comunicación entre los diferentes integrantes del Sistema de Seguridad Social en Salud y de las demás entidades que tengan a su cargo acciones en salud, en el marco de sus competencias y funciones.

- Facilitar la identificación apropiada de los recursos humanos, de infraestructura y financieros.

- Precisar las responsabilidades de los diferentes integrantes del Sistema de Seguridad Social en Salud y de las demás entidades que tengan a su cargo acciones en salud.

- Especificar resultados esperados de las intervenciones colectivas e individuales, dentro del proceso integral de la atención en salud.

Las rutas integrales de atención en salud tienen como objetivo garantizar la atención integral en salud a personas, familias y comunidades a partir de intervenciones de valoración integral de la salud, detección temprana, protección específica y diagnóstico. Abarcan, además, tratamiento, rehabilitación, paliación y educación para la salud, teniendo en cuenta el mejoramiento de la calidad en todo el continuo de atención, el logro de los resultados esperados en salud, la seguridad y el aumento de la satisfacción del usuario y la optimización del uso de los recursos ${ }^{18}$. En este caso, las clínicas y hospitales, las Entidades Administradoras de Planes de Beneficios de Salud y otras instituciones del Sistema General de Seguridad Social en Salud pueden ver las ventajas de esta modalidad en la cobertura y oportunidad como indicadores de calidad, integración y coordinación, e incluso en la seguridad del paciente; atributos que, a largo plazo, podrían constituir una disminución en los costos en salud ${ }^{22}$.

En la revisión de la literatura realizada no se hallaron referencias de rutas integrales de atención en salud en síndrome coronario agudo basadas en telesalud, pero se encontró que la experiencia de telesalud ha surgido como una estrategia que permite eliminar las distancias y las barreras en costos, mejorando el acceso a los servicios médicos. En Australia se halló que, al implementar una serie de acciones sistemáticas en la atención de los pacientes con síndrome coronario agudo, un $93.2 \%$ de dicha población tenían acceso oportuno a terapias de reperfusión (menos de 60 minutos para fibrinólisis y 120 minutos para intervención coronaria percutánea). Dentro de dichas acciones se encontraba la atención regionalizada de los casos bajo un modelo de telesalud georreferenciado, con interoperabilidad de los actores del sistema de emergencias médicas y la transferencia interhospitalaria sistemática desde unidades con acceso a fibrinólisis a otras con acceso a intervención coronaria percutánea ${ }^{23}$. Así mismo, la Sociedad Italiana de Cardiología respalda la utilización de la telemedicina en los casos de síndrome coronario agudo, dado que permite realizar un diagnóstico oportuno y mejorar el tiempo hasta el tratamiento, con una reducción de hasta un $50 \%$ en el tiempo desde el diagnóstico hasta el tratamiento en pacientes evaluados con telemedicina en comparación con aquellos que no lo fueron ${ }^{24}$. En el Estado de Nuevo León, México, el gobierno local implementó la telesalud como estrategia para mejorar la oportunidad en la atención a los pacientes que consultan a los servicios de urgencias hospitalarias en áreas rurales donde no se dispone de personal especializado, logrando aumentar la frecuencia de consulta con personal especializado y disminuyendo la frecuencia de traslados de los pacientes desde lugares distantes a centros hospitalarios especializados en las grandes ciudades para resolver el caso ${ }^{25}$.

Los pacientes con síndrome coronario agudo pueden generar incertidumbre diagnóstica o terapéutica, siendo susceptibles de atención por telesalud. Uno de los puntos clave en el manejo del paciente con síndrome coronario agudo e infarto de miocardio es la identificación de isquemia miocárdica ${ }^{26,27}$. Dichos hallazgos se hacen evidentes en la anamnesis y en el análisis y la interpretación del electrocardiograma ${ }^{28}$. La literatura científica ha reportado dificultades en la interpretación del electrocardiograma, en especial por la variabilidad interobservador, la cual se explica por el nivel de formación del personal clínico y la experiencia en el manejo de los casos, que puede impactar en la toma de decisiones en los casos de infarto de miocardio, sobre todo en aquellos que requieren terapia de reperfusión ${ }^{29-31}$. De acuerdo con lo anterior, y aunque no contamos con estudios en nuestro medio, este problema podría ser representativo en regiones distantes de centros hospitalarios especializados, como las zonas rurales. Con base en ello, el American College of Emergency Physicians avala la utilización de la telemedicina para la atención de pacientes urgentes, y sugiere que puede ofrecerse al personal médico y de 
enfermería, ubicado en lugares remotos y con incertidumbre en el manejo de los pacientes, siempre y cuando se cuente con la asesoría de personal con entrenamiento en emergencias ${ }^{32}$. Por lo anterior, se infiere que los modelos de ruta integral de atención en salud basados en telesalud, bajo guía de personal especializado, serían de gran ayuda para tomar decisiones más acertadas y oportunas 23,24 .

También deben mencionarse las barreras que limitan el desarrollo articulado entre los actores del sistema de salud para trabajar en una ruta integral, generando limitaciones de tipo investigativo, económico y político para su operación ${ }^{12}$. Algunas de estas barreras son:

- Exceso de autonomía de cada institución prestadora de servicios de salud.

- Segmentación de la población en varias aseguradoras, multiplicidad de contratos con diferentes operadores.

- Falta de articulación de los planes de beneficios y su operación en el ámbito local.

- Bajo nivel de coordinación y acción intersectorial por la salud.

- Ausencia de mecanismos de participación y planificación en salud.

- Debilidad en el acompañamiento territorial para la implementación de la política de atención integral en salud y del modelo integral de atención en salud.

- La habilitación de la red de servicios de las empresas promotoras de salud se hace sin tener en cuenta la capacidad de oferta de los prestadores presentes en los territorios.

- Baja integración de las tecnologías de la información y de la comunicación como modelos de desarrollo de plataformas tecnológicas.

- Escaso desarrollo de sistemas de referencia y contrarreferencia.

- Falta de intereses comunes de los actores en salud presentes en los territorios.

Por lo mencionado, con la implementación de la ruta integral de atención en salud para la atención de los pacientes con síndrome coronario agudo se pretende alcanzar objetivos claros, que a su vez permitan su adecuado desarrollo y evaluación. Los objetivos propuestos son:

- Mejorar la capacidad de respuesta y resolución del personal médico y paramédico en los servicios de urgencias donde son atendidos los pacientes con síndrome coronario agudo.

- Desarrollar mecanismos de comunicación y monitorización en tiempo real que permitan el enlace del centro remisor con el centro hospitalario especializado, para dirigir el caso como «paciente virtual» desde el inicio hasta el final de la ruta integral de atención en salud.

- Aumentar la frecuencia de utilización de medicamentos fibrinolíticos para la reperfusión en el síndrome coronario agudo en los primeros y segundos niveles de atención.

- Disminuir la morbilidad y la mortalidad de los pacientes con síndrome coronario agudo.

- Disminuir la carga de enfermedad relacionada con el síndrome coronario agudo en el sistema de salud.

- Disminuir los años de vida potencialmente perdidos de la población con síndrome coronario agudo.

- Emplear nuevas tecnologías para la prestación de servicios de salud, en el caso de la población con síndrome coronario agudo.

\section{Conclusiones}

Se cuenta con evidencia del efecto positivo de la telesalud al mejorar la oportunidad de la atención de los pacientes con síndrome coronario agudo. Aunque en nuestro país no se ha medido, esperamos que al integrar la telesalud a la ruta integral de atención en salud su efecto sea superior. Sin embargo, se requerirán investigaciones posteriores, tanto en lo conceptual como en el desarrollo de instrumentos, para su implementación y evaluación. Estas propuestas deben entenderse como un proceso y no como una meta ${ }^{33}$.

Las brechas en conectividad y en el uso de las TIC, la educación médica continuada y el compromiso y el aporte por parte de directores y líderes de procesos institucionales en el sector salud a este problema, son los tres retos identificados más importantes para la implementación de este tipo de modelos, y deben ser abordados de manera integral para lograr un impacto.

\section{Agradecimientos}

Los autores agradecen a los doctores Tricel Melisa Villegas Zuluaga, especialista en medicina de urgencias, y Juan Manuel Senior Sánchez, cardiólogo intervencionista, por su invaluable aporte al desarrollo conceptual de esta ruta integral de atención en salud.

A especialistas de medicina de urgencias de la Universidad de Antioquia, Luisa María Escobar del Río y Juan Manuel Robledo Cadavid, por su aporte en el desarrollo del manual de esta ruta integral de atención en salud.

Al Sistema General de Regalías por el apoyo financiero al programa de telesalud de la Facultad de Medicina de la Universidad de Antioquia. 


\section{Conflicto de intereses}

Los autores de este artículo no tienen ningún conflicto de intereses por declarar.

\section{Financiamiento}

Facultad de Medicina, Universidad de Antioquia, a través del Posgrado de Medicina de Urgencias, y Grupo de Investigación en Urgencias y Emergencias (GIURE).

\section{Responsabilidades éticas}

Protección de personas y animales. Los autores declaran que para esta investigación no se han realizado experimentos en seres humanos ni en animales.

Confidencialidad de los datos. Los autores declaran que en este artículo no aparecen datos de pacientes.

Derecho a la privacidad y consentimiento informado. Los autores declaran que en este artículo no aparecen datos de pacientes.

\section{Bibliografía}

1. Writing Group Members; Mozaffarian D, Benjamin EJ, Go AS, Arnett DK, Blaha MJ, Cushman M, et al. Heart Disease and Stroke Statistics 2016 Update: A Report From the American Heart Association. Circulation. 2016;133:e38-360.

2. GBD 2017 Disease and Injury Incidence and Prevalence Collaborators Global, regional, and national incidence, prevalence, and years lived with disability for 354 diseases and injuries for 195 countries and territories, 1990-2017: a systematic analysis for the Global Burden of Disease Study 2017. Lancet. 2018;392:1789-858.

3. Instituto Nacional de Salud de Colombia. Carga de enfermedades crónicas no transmisibles y discapacidad en Colombia. Inf Técnico Obs Nac Salud. 2015; V Edición.

4. Ministerio de Salud y Protección Social. Analisis de la situación en salud. Colombia 2015. Bogotá; 2015. Disponible en: https://www.minsalud.gov. $\mathrm{co} / \mathrm{sites} / \mathrm{rid} /$ Lists/BibliotecaDigital/RIDE/VS/ED/PSP/asis-2015.pdf.

5. Ministerio de Salud y Protección Social, Colciencias, Universidad de Antioquia. Guía de práctica clínica para el síndrome coronario agudo. Bogotá; 2013. Disponible en: http://gpc.minsalud.gov.co/gpc_sites/Repositorio/Conv_500/GPC_s_coronario/GPC_Comple_SCA.pdf

6. The Task Force on the management of ST-segment elevation acute myocardial infarction of the European Society of Cardiology (ESC); Steg PG, James SK, Atar D, Badano LP, Blömstrom-Lundqvist C, Borger MA, et al. ESC Guidelines for the management of acute myocardial infarction in patients presenting with ST-segment elevation. Eur Heart J. 2012;33:2569-619.

7. Gara PTO, Kushner FG, Ascheim DD, Casey DE Jr, Chung MK, de Lemos JA et al. 2013 ACCF/AHA Guideline for the management of ST-elevation myocardial infarction: a report of the American College of Cardiology Foundation/American Heart Association Task Force on Practice Guidelines. Circulation. 2013;127:e362-425

8. Ibanez B, James S, Agewall S, Antunes MJ, Bucciarelli-Ducci C, Bueno $\mathrm{H}$, et al. 2017 ESC Guidelines for the management of acute myocardial infarction in patients presenting with ST-segment elevation: The Task Force for the management of acute myocardial infarction in patients presenting with ST-segment elevation of the European Society of Cardiology (ESC). Eur Heart J. 2018;39:119-77.

9. Roffi M, Patrono C, Collet JP, Mueller C, Valgimigli M, Andreotti F, et al. 2015 ESC Guidelines for the management of acute coronary syndromes in patients presenting without persistent ST-segment elevation: Task force for the management of acute coronary syndromes in patients presenting without persistent ST-segment elevation of the European Society of Cardiology (ESC). Eur Heart J. 2016;37:267-315.
10. Amsterdam EA, Wenger NK, Brindis RG, Casey DE Jr, Ganiats TG, Holmes DR Jr, et al. 2014 AHA/ACC Guideline for the management of patients with non-ST-elevation acute coronary syndromes: executive summary: a report of the American College of Cardiology/American Heart Association Task Force on Practice Guidelines. Circulation. 2014;130:2354-94.

11. Ayala-García J. La salud en Colombia: más cobertura pero menos acceso. 2012. Disponible en: https://www.banrep.gov.co/sites/default/files/ publicaciones/archivos/dtser_204.pdf.

12. Restrepo-Zea JH, Silva-Maya C, Andrade-Rivas F, Vh-Dover R. Acceso a servicios de salud: análisis de barreras y estrategias en el caso de Medellín, Colombia. Rev Gerenc Polit Salud. 2014;13:236-59.

13. Ojeda DM, Ojeda DM, Social S. Plata hay, la falla del Sistema de Salud en Colombia es estructural. Asoc Colomb Clínicas y Hosp. 2012:34-36. Disponible en: http://www.achc.org.co/documentos/hospitalaria/La entrevista 82.pdf.

14. Ministerio de la Protección Social. Ley 1751 del 2015. Colombia; 2015. Disponible en: http://www.minsalud.gov.co/Normatividad_Nuevo/Ley 1751 de 2015.pdf.

15. Ministerio de Salud y Protección Social. República de Colombia. Integralidad en la atención es fundamental en el MIAS. 2016. Disponible en: https://www.minsalud.gov.co/Paginas/Integralidad-en-la-atencion-es-fundamental-en-el-MIAS.aspx.

16. Ministerio de Salud y Protección Social. Resolución 0429 del 2016. Disponible en: https://www.minsalud.gov.co/Normatividad_Nuevo/Resolución 0429 de 2016.pdf.

17. Congreso de la Republica de Colombia. Ley 1419 de 2010. Colombia; 2010. Disponible en: http://wsp.presidencia.gov.co/Normativa/Leyes/Documents/ley141913122010.pdf.

18. Ministerio de Salud y Protección Social. Manual metodológico para la elaboración e implementación de las RIAS. 2016. Disponible en: https:// www.minsalud.gov.co/sites/rid/Lists/BibliotecaDigital/RIDE/VS/Manual-metodologico-rias.pdf.

19. Ministerio de Salud y Protección Social de Colombia. Resolución No. 2654 del 2019. Colombia; 2019. Disponible en: https://www.minsalud.gov.co/Normatividad_Nuevo/Resolución No. 2654 del 2019.pdf.

20. Ministerio de Salud y Protección Social. Nuevo marco reglamentario para la telesalud en Colombia. Disponible en: https://www.minsalud.gov.co/ sites/rid/Lists/BibliotecaDigital/RIDE/DE/OT/nuevo-marco-reglamentario-para-la-telesalud-en-colombia-18122019.pdf.

21. Colorado Vélez A, Gómez Granada J, Elorza Saldarriaga JF, Cárdenas Velásquez N, García Cano JF, Ossa Estrada DA, et al. Gestión territorial de la salud: perspectivas, aprendizajes y aportes a la práctica. Universidad de Antioquia, ed. Revista de la Facultad Nacional de Salud Pública. 2017;35:202. https://revistas.udea.edu.co/index.php/fnsp/article/view/328206.

22. Organización Panamericana de la Salud. Redes integradas de servicios de salud. 2010. Disponible en: https://www.paho.org/uru/index.php?option=com_docman\&view=download\&alias=145-redes-integradas-de-servicios-de-salud-aps-n4\&category_slug=publicaciones-sistemas-y-servicios-de-salud\&ltemid=307.

23. Ranasinghe I, Turnbull F, Tonkin A, Clark RA, Coffee N, Brieger D. Comparative effectiveness of population interventions to improve access to reperfusion for ST-segment - elevation myocardial infarction in Australia. Circ Cardiovasc Qual Outcomes. 2012;5:429-36.

24. Caldarola P, Gulizia MM, Gabrielli D, Sicuro M, De Gennaro L, Giammaria M, et al. ANMCO/SIT Consensus Document: Telemedicine for cardiovascular emergency networks. Eur Heart J Suppl. 2017;19(Suppl D):D229-43.

25. González ML, Adrián R, López P. Desarrollo de la telesalud en México. México, DF; 2013. Disponible en: https://repositorio.cepal.org/bitstream/ handle/11362/4055/1/S2012935_es.pdf.

26. Sandoval $\mathrm{Y}$, Thygesen $\mathrm{K}$, Jaffe AS. The universal definition of myocardial infarction: present and future. Circulation. 2020;141:1434-6.

27. Thygesen $\mathrm{K}$, Alpert JS, Jaffe AS, Chaitman BR, Bax JJ, Morrow DA et al. Fourth universal definition of myocardial infarction (2018). Circulation. 2018;138:e618-51.

28. Birnbaum Y, Wilson JM, Fiol M, de Luna AB, Eskola M, Nikus K. ECG diagnosis and classification of acute coronary syndromes. Ann Noninvasive Electrocardiol. 2014;19:4-14.

29. Mccabe JM, Armstrong EJ, Ku I, Kulkarni A, Hoffmayer KS, Bhave PD, et al. Physician accuracy in interpreting potential ST-segment elevation myocardial infarction electrocardiograms. J Am Heart Assoc. 2013;2:1-9.

30. Massel D. Observer variability in ECG interpretation for thrombolysis eligibility : experience and context matter. J Thromb Thrombolysis. 2003;15:131-40.

31. Pines JM, Perina DG, Brady WJ. Electrocardiogram interpretation training and competency assessment in emergency medicine residency programs. Acad Emerg Med. 2004;11:982-4.

32. American College of Emergency Physicians. Emergency Medicine Telemedicine Originally. United States; 2016. Disponible en: https://www. acep.org/globalassets/new-pdfs/policy-statements/emergency-medicine-telemedicine.pdf.

33. López Puig P, Morales Suárez I del R, Lara Menchaca S, Martínez Trujillo N, Lau López S, Soler Cárdenas SF. Las redes integradas de servicios de salud desde la realidad cubana. Rev Cuba Salud Publica. 2009;35:34-43. 